\title{
A strategic framework improves access to stroke reperfusion across the state of Victoria,
}

Australia.

Janet E. Bray PhD, ${ }^{1}$ Sonia Denisenko MPH, ${ }^{2}$ Bruce C.V. Campbell MBBS PhD, ${ }^{3}$ Michael Stephenson BHlthSc, Grad Dip (MICA), ${ }^{4}$ Jason Muller GIS Analyst, ${ }^{4}$ Grant Hocking Grad Dip (Ed), ${ }^{4}$ Peter J. Hand MD, ${ }^{2,3}$ Christopher F. Bladin MD ${ }^{5,6}$

${ }^{1}$ Department of Epidemiology and Preventive Medicine, Monash University, Victoria, Australia

${ }^{2}$ Victorian Stroke Clinical Network, Department of Health and Human Services, Victoria, Australia

${ }^{3}$ Department of Medicine and Neurology, Melbourne Brain Centre, Royal Melbourne Hospital and University of Melbourne, Victoria, Australia

${ }^{4}$ Ambulance Victoria, Doncaster, Victoria, Australia

${ }^{5}$ The Florey Institute of Neuroscience and Mental Health, Melbourne, Victoria, Australia

${ }^{6}$ Eastern Health Clinical School, Monash University, Melbourne, Victoria, Australia

Corresponding author: Dr Janet Bray, Department of Epidemiology and Preventive Medicine, Monash University, Level 5, The Alfred Centre, Melbourne, Victoria, Australia. Telephone: +61399030177 Email: janet.bray@monash.edu

\section{Acknowledgements}

VST program acknowledges financial support from the Victorian Stroke Clinical Network, Monash University, Boehringer Ingelheim. Drs Bray and Campbell are funded by National Health and Medical Research Council (NHMRC) and Heart Foundation (HF) Fellowships (JB: NHMRC \#1069985 and HF \#100136; BC: NHMRC \#1111972 and HF \#100782).

This is the author manuscript accepted for publication and has undergone full peer review but has not been through the copyediting, typesetting, pagination and proofreading process, which may lead to differences between this version and the Version of Record. Please cite this article as doi: $10.1111 / \mathrm{imj} .13494$

This article is protected by copyright. All rights reserved. 
Abstract count: 229

Word Count: 2848

This article is protected by copyright. All rights reserved. 
The best outcomes for acute ischaemic stroke patients are achieved with rapid access to hospitals offering stroke thrombolysis and endovascular thrombectomy. However, the availability of these services can vary, particularly in regional and rural areas.

For example, in 2010 in the Australian state of Victoria, we found that only $65 \%$ of suspected acute stroke patients in regional areas were transported to thrombolysis centres (compared to $89 \%$ of metropolitan patients) ${ }^{1}$. When we examined the location of these regional patients, we found large regions of the state had no thrombolysis centres or had long transport times to receive treatment ( $23 \%$ of rural patients were located outside a 60 -minute drive-time $)^{2}$.

In response, and more recently in planning for the implementation of a state-wide endovascular thrombectomy service, key stakeholders (Victorian Stroke Clinical Network, Department of Health and Human Services, Victorian Stroke Telemedicine Program and local health services) developed strategies to improve state-wide access (Table 1), including telemedicine services and "Drip and Ship" protocols ${ }^{2-4}$. This study aims to examine the impact of these strategies and whether access by road transport to acute stroke care has improved.

\section{Methods}

\section{Setting}

The study took place in the Australian state of Victoria, which is located in the southeast of Australia and has an area size of $227,416 \mathrm{~km}$. The 2015 population was 5.96 million, of whom $24 \%$ reside outside the greater metropolitan city of Melbourne. This study was approved by the Monash University Human Ethics Committee (CF16/2095 - 2016001041). 
We used data from Ambulance Victoria for all patients attended by ambulance with suspected stroke (Medical Dispatch Priority System Code 28) for the period November $1^{\text {st }}$ 2014 to October $31^{\text {st }} 2015$. Ambulance Victoria are the sole provider of emergency medical services across the whole of Victoria.

\section{Major changes to regional stroke services}

Three major changes have occurred in Victorian regional stroke services in the last decade.

First, in response to the release of the Stroke Care Strategy for Victoria, a specialist Stroke Project Manager and Regional Coordinators were employed to implement the recommendations of the Strategy ${ }^{5}$. Outcomes from this process were: the development of new stroke units in seven regional hospitals and the delivery of the Framework for regional acute stroke services in Victoria -a strategic document which guides the transport of suspected stroke patients to hospitals with stroke service capability ${ }^{2}$.

Second, the Victorian Stroke Telemedicine (VST) Program ${ }^{4}$ was piloted $^{6}$ and implemented across the state. The VST currently provides a 24-hour clinical service for acute stroke care to 16 regional hospitals. Patients are assessed by video link to a stroke neurologist, assessed for eligibility for thrombolysis which is then administered if appropriate. In the majority of cases, patients are then admitted to the hospitals stroke unit. Two sites without stroke unit capability have formal protocols in place to transfer thrombolysed patients to the nearest hospital with stroke unit access (“Drip and ship"). 
Third, the delivery of ECR therapy commenced. To do this a ECR Steering Committee was formed and developed a state-wide service $\operatorname{protocol}^{3}$. The protocol includes the: 1) criteria for ECR centres; 2) protocols to facilitate the transfer (of relevant patients) from VST supported sites to metropolitan ECR centres; and 3) the clinical decision-making for the delivery of care from symptom onset to management post-ECR. Eligibility to be a state wide ECR provider included the ability to provide a 24-hour service, neurointervention capabilities and meeting the Australian requirements of a comprehensive stroke service. The Steering Committee decided the final number and location of the two ECR centres taking into account eligibility criteria, training, case load size and expression of interest applications. Three additional sites in the metropolitan area provide ECR to patients that arrive from their local catchment, but do not receive regional patients.

\section{Analysis and mapping}

The location of the patients and reperfusion centres (regional VST sites, metropolitan thrombolysis sites and endovascular thrombectomy centres) were individually mapped (via longitude and latitude), and drive-times to reperfusion centres were computed using MapInfo (MapInfo Drivetime®, Pitney Bowes Inc.). Centres offering thrombolysis were identified through the Stroke Foundation Website ${ }^{7}$. Currently in Victoria there is one centre receiving regional patients for endovascular thrombectomy, with another centre commencing in 2017. The proportion of regional patients within 1) 60-minutes drive-time to centres offering thrombolysis and 2) 180-minutes of the two endovascular thrombectomy centres were calculated. A statistical comparison to the 60 -minute 2010 data was made using the Chisquare statistic ${ }^{2}$ 


\section{Results}

In the 1-year study period, paramedics at Ambulance Victoria attended 16,418 Code 28 cases ( $2.9 \%$ of all emergency calls), of whom 4597 (28\%) were located in regional Victoria.

Between 2010 and 2015, a significant increase was seen in the proportion of suspected stroke patients within a 60-minute drive-time to a thrombolysis centre in both regional Victoria ( $77 \%$ to $95 \%, \mathrm{p}<0.001)$ and the entire state $(89 \%$ to $99 \%, \mathrm{p}>0.001)$. To illustrate changes in access, we mapped the 2015 cases and overlayed the 60-minute drive times from 2010 and 2015 (Figure 1).

In the 2015 data, the majority of suspected stroke patients were located within a 3-hour drivetime to the two designated ECR providers ( $88 \%$ of regional patients and $96 \%$ for entire state).

\section{Discussion}

Our study indicates access to reperfusion therapies by road ambulance is now possible for the majority of stroke patients in the Australian state of Victoria. A strategic, region-specific approach has seen improvements in rapid access to stroke thrombolysis -with 95\% of regional Victoria now within 60-minutes by road transport. Furthermore, access to ECR by road transportation will be feasible for the vast majority (96\%) of Victorians with the addition of a second receiving site in 2017.

Access by road to thrombolysis centres in our population is now significantly higher than rates reported in other countries; although they too have reported improvements in access. For example, in the United States (US) it was recently estimated that $87 \%$ of the population 
were within 60 -minutes of a thrombolysis centre by ground transport ${ }^{8}$, an increase from $55 \%$ in $2008^{9}$ and $63 \%{ }^{10}$ in 2010 . However, this recent report also found significant regional variation between US states $(33 \% \text { to } 100 \%)^{8}$. Part of this variation in access is likely related to geographical access. For example, areas in our state that currently fall outside the 60minute road transport time have sparse populations (typically 0.7 persons $/ \mathrm{km}^{2}$ ), no large regional towns and are in either mountainous regions or regions classified as remote. Ambulance Victoria provide air transport in these regions, but this is dependent on availability and weather.

Ensuring an entire population can receive equitable acute stroke services requires considerable resources across a health care system. In our region this was enabled and facilitated through the Victorian Department of Health and Human Services via its Stroke Clinical Network. The Network developed a strategic framework ${ }^{2}$, worked in consultation with local health services to develop region specific protocols and procedures, and funded regional stroke coordinators. The Australian government funded the expansion of the Victorian Stroke Telemedicine Program to encompass 16 sites across the state. These initiatives have established a coordinated regional referral framework that enables patients with suspected stroke to be transported to an appropriate local hospital, have a teleconsultation with a stroke specialist and, if appropriate, receive reperfusion therapies including referral for ECR and other advanced therapies. The next phase of this program is to fully implement the state-wide service protocol for ECR for acute stroke . $^{3}$

These initiatives are likely to have contributed to the high thrombolysis rate observed in Victoria, which was $11 \%$ in 2015 and compares favourably to the national Australian average 
of $7 \%{ }^{11}$. We also note an increase in thrombolysis rates across regional Victoria in hospitals contributing data to the Stroke Foundation's Acute Stroke Audit ${ }^{11,12}$ (from 6\% in 2011 to 13\% in 2015 -personal correspondence from the Stroke Foundation). These results, which must be interpreted with caution as few regional hospitals provide data to the audit (9 in 2011 and 5 in 2015), are supported by results from the Victorian Stroke Telemedicine program which also reported an increase in thrombolysis and a decrease in door-to-needle times in regional hospitals. ${ }^{6}$

This study has a number of limitations -which mainly relate to the use of ambulance data. First, our study did not examine access in patients who present to hospital via private vehicles (approximately $20 \%$ of Australian strokes ${ }^{13}$ ). It is possible that some of these cases may differ in access to reperfusion services to our study population and this requires future study. Second, we used a dispatcher diagnosis of suspected stroke, and not paramedic or hospital diagnosis, as this data was readily available and contains location coordinates. The sensitivity of this data is unknown, but previous data using a similar call taking system suggests approximately $83 \%$ of these patients have a final diagnosis of stroke ${ }^{14}$. Third, due to the remoteness of some regions we did not use average drive-times derived from population data, such as the centre point of census tracts as used in previous reports ${ }^{8}$. Therefore, our data are only estimates of access to reperfusion services. Lastly, we are unable to report patient outcomes, which is needed to completely understand the impact of these strategies.

\section{Conclusion}


In summary, a strategic approach has resulted in improved access by road transport to stroke reperfusion therapies across the Australian state of Victoria, achieving a very high $(>90 \%)$ level of consistency across regional and metropolitan populations.

\section{Acknowledgements}

VST program acknowledges financial support from the Victorian Stroke Clinical Network, Monash University, Boehringer Ingelheim. Drs Bray and Campbell are funded by National Health and Medical Research Council (NHMRC) and Heart Foundation (HF) Fellowships (JB: NHMRC \#1069985 and HF \#100136; BC: NHMRC \#1111972 and HF \#100782).

\section{Conflicts of interest}

Ms Denisenko is employed by the Department of Health and Human Services.

\section{References}

1 Bray JE, Coughlan K, Mosley I, Barger B, Bladin C. Are suspected stroke patients identified by paramedics transported to appropriate stroke centres in Victoria, Australia? Intern Med J. 2014; 44: 515-8.

2 Victorian Stroke Clinical Network (Department of Health). Framework for regional acute stroke services in Victoria. https://www2healthvicgovau/hospitals-and-healthservices/quality-safety-service/clinical-networks/clinical-network-stroke/stroke-statewideframeworks. 2013: Last Accessed 19/9/2016.

3 Victorian Stroke Clinical Network (Department of Health). Endovacular clot retrieval for acute stroke: Statewide service protocol for Victoria. . https://www2healthvicgovau/hospitals-and-health-services/quality-safety-service/clinical-

This article is protected by copyright. All rights reserved. 
networks/clinical-network-stroke/stroke-statewide-frameworks 2015: Last accessed: $19 / 9 / 2016$

4 Cadilhac DA, Moloczij N, Denisenko S, Dewey H, Disler P, Winzar B, et al. Establishment of an effective acute stroke telemedicine program for Australia: protocol for the Victorian Stroke Telemedicine project. International journal of stroke : official journal of the International Stroke Society. 2014; 9: 252-8.

$5 \quad$ Victorian Stroke Clinical Network (Department of Health). Stroke Care Strategy Victoria. https://www2healthvicgovau/hospitals-and-health-services/quality-safetyservice/clinical-networks/clinical-network-stroke/stroke-care-strategy. 2007; Last Accessed 19/9/2016. .

6 Bladin CF, Molocijz N, Ermel S, Bagot KL, Kilkenny M, Vu M, et al. Victorian Stroke Telemedicine Project: implementation of a new model of translational stroke care for Australia. Internal Medicine Journal. 2015; 45: 951-56.

7 National Stroke Foundation. Stroke Care Units. https://strokefoundationcomau/aboutstroke/treatment-for-stroke/stroke-care-units. 2016; Last Accessed: 19/09/2016.

8 Adeoye O, Albright KC, Carr BG, Wolff C, Mullen MT, Abruzzo T, et al. Geographic access to acute stroke care in the United States. Stroke; a journal of cerebral circulation. 2014; 45: 3019-24.

9 Albright KC, Branas CC, Meyer BC, Matherne-Meyer DE, Zivin JA, Lyden PD, et al. ACCESS: acute cerebrovascular care in emergency stroke systems. Archives of neurology. 2010; 67: 1210-8.

This article is protected by copyright. All rights reserved. 
10 Mullen MT, Branas CC, Kasner SE, Wolff C, Williams JC, Albright KC, et al.

Optimization modeling to maximize population access to comprehensive stroke centres.

Neurology. 2015; 84: 1196-205.

11 National Stroke Foundation. National Stroke Audit - Acute Services Report 2015. https://strokefoundationcomau/en/What-we-do/Treatment-programs/Stroke-datacollection/Acute-audit. 2015: Last accessed: 19/09/2016.

12 National Stroke Foundation. National Stroke Audit - Acute Services Organisational Survey Report 2011. . http://strokefoundationcomau/site/media/NSF_Audit-

Report_2011_web4pdf. 2011: Last Accessed 10/2012.

13 National Stroke Foundation. National Stroke Audit - Acute Services Clinical Audit Report 2013. Melbourne, Australia 2013.

14 Ramanujam P, Guluma KZ, Castillo EM, Chacon M, Jensen MB, Patel E, et al. Accuracy of stroke recognition by emergency medical dispatchers and paramedics--San Diego experience. Prehospital emergency care : official journal of the National Association of EMS Physicians and the National Association of State EMS Directors. 2008; 12: 307-13. 15 Victorian Stroke Clinical Network (Department of Health). Regional stroke transfer framework -Barwon-South Western Health region. https://www2healthvicgovau/hospitalsand-health-services/quality-safety-service/clinical-networks/clinical-network-stroke/strokestatewide-frameworks. 2015: Last accessed: 19/9/2016.

16 Victorian Stroke Clinical Network (Department of Health). Lodden Mallee regional frameword for managing acute stroke. https://www2healthvicgovau/hospitals-and-health-

This article is protected by copyright. All rights reserved. 
services/quality-safety-service/clinical-networks/clinical-network-stroke/stroke-statewideframeworks. 2016: Last Accessed 19/9/2016.

17 Cadilhac DA, Lannin NA, Anderson CS, Levi CR, Faux S, Price C, et al. Protocol and pilot data for establishing the Australian Stroke Clinical Registry. International journal of stroke : official journal of the International Stroke Society. 2010; 5: 217-26.

\section{Figure Legend}

Figure 1: Access by 60-minute drive-time (shaded areas) for suspected stroke patients (blue dots) to stroke thrombolysis centres (red crosses) in 2010 (top) and 2015 (bottom).

This article is protected by copyright. All rights reserved. 
Table 1. Strategies used in the improvement for regional access to reperfusion therapies.

\begin{tabular}{|c|c|c|}
\hline Strategies and Initiatives & Key Stakeholders & Outcomes \\
\hline Stroke Care Strategy $2007^{5}$ & $\begin{array}{l}\text { Victorian Department of Health Stroke Clinical } \\
\text { Network }\end{array}$ & $\begin{array}{l}\text { Implementation of acute stroke services - } \\
\text { specifically stroke unit development via the } \\
\text { appointment of state-wide stroke project manager } \\
\text { and regional facilitators }\end{array}$ \\
\hline $\begin{array}{l}\text { Framework for regional acute stroke } \\
\text { services in Victoria } 2013^{2} \\
\text { Release of subsequent region specific } \\
\text { stroke referral frameworks released } \\
2015^{15}, 2016^{16}\end{array}$ & $\begin{array}{l}\text { Victorian Department of Health Stroke Clinical } \\
\text { Network } \\
\text { Local stakeholder sites } \\
\text { Departments of Health Regional offices } \\
\text { Ambulance Victoria }\end{array}$ & $\begin{array}{l}\text { Development of referral pathways to ensure } \\
\text { appropriate transfer of suspected stroke patients to } \\
\text { regional acute stroke units. } \\
\text { Ambulance Victoria protocol for stroke patient } \\
\text { transport stroke services (including bypass protocols } \\
\text { for smaller sites) } \\
\text { Increase awareness of available stroke services } \\
\text { across local systems and transfer guidelines }\end{array}$ \\
\hline $\begin{array}{l}\text { Victorian Stroke Telemedicine } \\
\text { program pilots - Bendigo and Loddon } \\
\text { Mallee expansion }\end{array}$ & $\begin{array}{l}\text { Victorian government funding } \\
\text { Florey Institute of Neuroscience and Mental Health } \\
\text { Victorian Department of Health Stroke Clinical } \\
\text { Network }\end{array}$ & $\begin{array}{l}\text { Delivery of specialist advice services to Bendigo } \\
\text { Hospital (regional hub site) and subregional services } \\
\text { (smaller hospitals) within one area (Loddon Mallee) } \\
\text { of regional Victoria }\end{array}$ \\
\hline
\end{tabular}

This article is protected by copyright. All rights reserved. 


\begin{tabular}{|c|c|c|}
\hline & $\begin{array}{l}\text { Ambulance Victoria } \\
\text { Stroke Foundation }\end{array}$ & \\
\hline $\begin{array}{l}\text { Victorian Stroke Telemedicine State } \\
\text { wide program }\end{array}$ & $\begin{array}{l}\text { Australian Government funding } \\
\text { Florey Institute of Neuroscience and Mental Health } \\
\text { Victorian Department of Health Stroke Clinical } \\
\text { Network } \\
\text { Ambulance Victoria } \\
\text { Stroke Foundation }\end{array}$ & $\begin{array}{l}\text { State wide implementation of Stroke telemedicine } \\
\text { service }{ }^{6} \text {, and monitoring via the Australian Stroke } \\
\text { Clinical Registry (AuSCR) }^{17}\end{array}$ \\
\hline $\begin{array}{l}\text { Development of Endovascular Clot } \\
\text { retrieval protocol } 2016\end{array}$ & $\begin{array}{l}\text { Victorian Department of Health and Human } \\
\text { Services Stroke Clinical Network } \\
\text { Metropolitan and Regional Hospitals } \\
\text { Victorian Stroke Telemedicine Program } \\
\text { Ambulance Victoria }\end{array}$ & $\begin{array}{l}\text { Two major tertiary ECR centres located on opposite } \\
\text { sides of the major metropolitan area } \\
\text { Monitoring via AuSCR } \\
\text { Victorian Stroke Telemedicine program to triage } \\
\text { suitable ECR patients } \\
\text { Ambulance Victoria protocol to transport } \\
\text { Regional health services to retrieve patients post } \\
\text { ECR intervention once stable }\end{array}$ \\
\hline
\end{tabular}

This article is protected by copyright. All rights reserved. 


\begin{abstract}
Background: In 2010, rapid access to stroke thrombolysis centres was limited in some regional areas in the Australian state of Victoria. These results, and in planning for Endovascular Clot Retrieval (ECR), have led to the implementation of strategies by the Victorian Stroke Clinical Network, the Victorian Stroke Telemedicine Program and local health services to improve state-wide access.
\end{abstract}

Aims: This study aims to examine whether access to stroke reperfusion services (thrombolysis and ECR) in regional Victoria have subsequently improved.

Methods: The location of suspected stroke patients attended by ambulance in 2015 were mapped and drive-times to the nearest reperfusion services were calculated. We then calculated the proportion of cases with transport times within: 1) 60-minutes to thrombolysis centres and 2) 180-minutes to two ECR centres designated to receive regional patients. Statistical comparisons to existing 2010 data were made.

Results: In 2015, Ambulance Victoria attended 16,418 cases of suspected stroke $(2.9 \%$ of all emergency calls), of whom 4597 (28\%) were located in regional Victoria. Compared to 2010, a greater proportion of regional suspected stroke patients in 2015 were located within 60minutes of a thrombolysis centre by road $(77 \%$ to $95 \%, \mathrm{p}<0.001)$. A 3 -hour road travel time to the two ECR centres is currently possible for $88 \%$ of regional patients.

Conclusions: A strategic and region-specific approach has resulted in improved access by road transport to reperfusion therapies for stroke patients across Victoria.

Keywords: Stroke, thrombolysis, health services

This article is protected by copyright. All rights reserved. 
Figure 1: Access by 60-minute drive-time (shaded areas) for suspected stroke patients (blue dots) to stroke thrombolysis centres (red crosses) in 2010 (top) and 2015 (bottom).
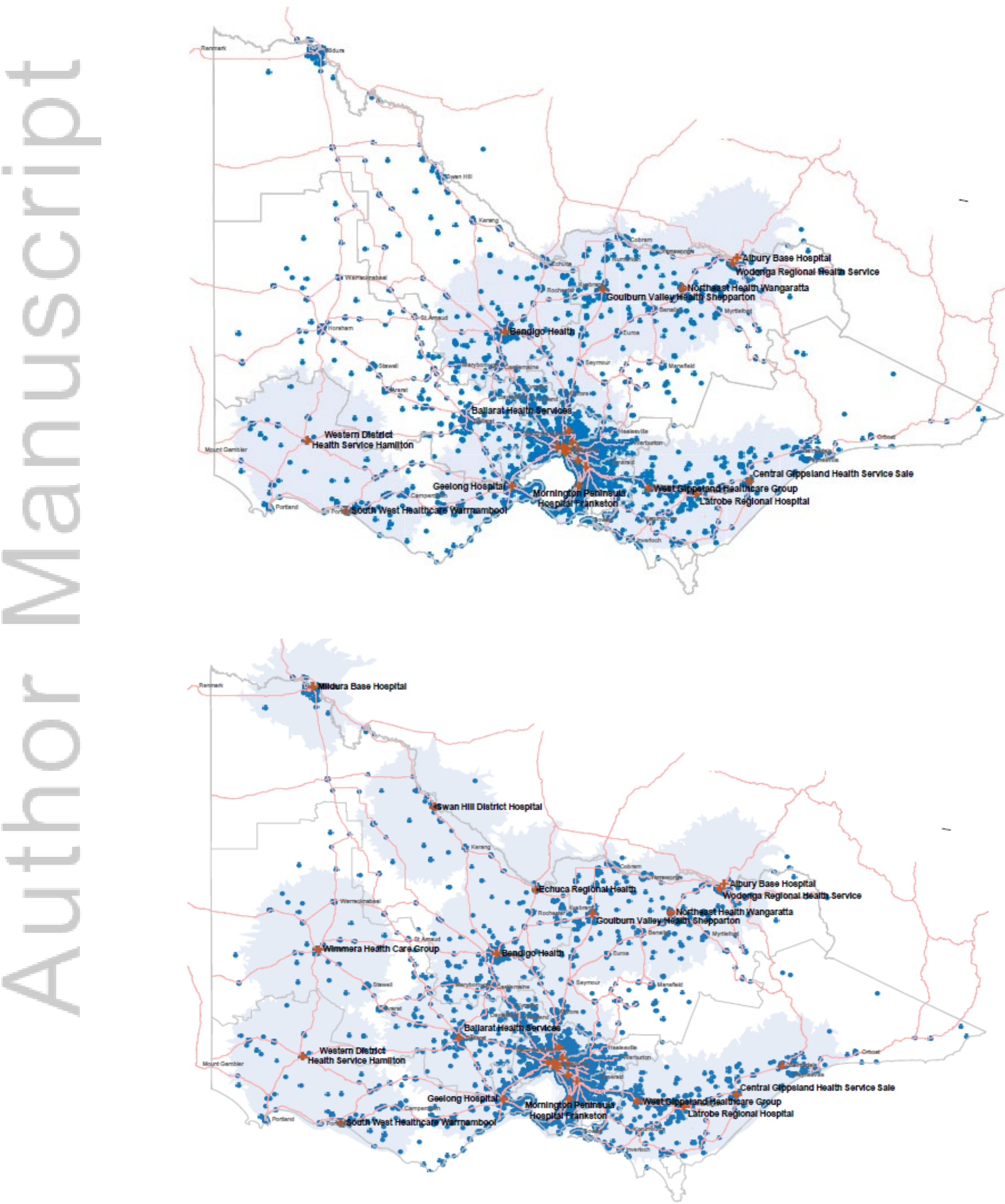

This article is protected by copyright. All rights reserved. 


\section{University Library}

\section{- M M I N E R VA A gateway to Melbourne's research publications}

Minerva Access is the Institutional Repository of The University of Melbourne

Author/s:

Bray, JE;Denisenko, S;Campbell, BCV;Stephenson, M;Muller, J;Hocking, G;Hand, PJ;Bladin, CF

Title:

Strategic framework improves access to stroke reperfusion across the state of Victoria Australia

Date:

2017-08-01

Citation:

Bray, J. E., Denisenko, S., Campbell, B. C. V., Stephenson, M., Muller, J., Hocking, G., Hand, P. J. \& Bladin, C. F. (2017). Strategic framework improves access to stroke reperfusion across the state of Victoria Australia. INTERNAL MEDICINE JOURNAL, 47 (8), pp.923-928. https://doi.org/10.1111/imj.13494.

Persistent Link:

http://hdl.handle.net/11343/293255 